\title{
Image Collection using an Auto Data Acquisition System and An Application to Ice embedded Ribosome
}

\author{
Yoshitaka Aoyama $^{1}$, Hideo Nishioka $^{2}$ and Yukihito Kondo ${ }^{3}$ \\ 1.JEOL, 1-2 Musashino 3-Chome Akishima Tokyo 196-8558 Japan
}

Single particle analysis is a tomographic method to reconstruct a three-dimensional structure from a set of two-dimensional projected images acquired by a transmission electron microscope (TEM). This method is applied to reconstruct biological samples such as proteins or viruses. The micrographs of cryo biological samples are obtained by using a minimum dose system (MDS), because these samples are sensitive to incident electrons. The low dose leads to bad signal noise ratio (SNR) of the particle images picked from the micrographs. In order to obtain a high contrast image of the particles, similar images picked from the micrographs are classified and merged by hierarchical ascendant classification, which requires many micrographs. Consequently, many images of the particle are necessary to reconstruct a three-dimensional structure by the single particles analysis.

Some research groups have developed the software to get micrographs from cryo-samples automatically for single particle analysis ${ }^{[1-3]}$. JEOL data acquisition system (JADAS) is one of the software developed in $2009^{[4]}$. Recently, we have improved the JADAS to be easy to use. In this paper, we report the threedimensional structure of ribosome using the new JADAS.

Figure 1 shows a GUI of the JADAS. The auto acquisition sequence is as follows. At first, the JADAS takes a low magnification image, which is shown as a Global map at the upper left of the GUI, and finds locations of holes in a sample mesh automatically. Next, the JADAS takes local maps at the position of these holes, as shown at the upper left of the GUI, and detects the grid holes of a Quantifoil. Finally, the JADAS automatically takes images at each hole with MDS mode as shown in the main panel of the GUI. The previously taken images are listed in the small icons in the lower panel. The information of cameras and a image shown in the main panel are shown panel between the main and global map panels. Functions for JADAS operation are listed at the upper of the GUI.

Ribosome prepared from E-coli was dropped on Quantifoil and ice-embedded by EM-GP (Leica). Thusmade sample grid was transferred to a field emission TEM (JEM-2100F) whose sample holder was kept at low temperature with liquid nitrogen. The images of the ribosome were automatically obtained by the new JADAS at $200 \mathrm{kV}$. An electron dose onto the sample was $10 \mathrm{e}^{-} / \mathrm{A}^{2}$. Magnification was $60,000 \mathrm{x}$. In our auto acquisition of ribosome, the JADAS took 36 images per hour. Using the JADAS, we could obtain images of the cryo specimen to reconstruct three-dimensional structure of ribosome.

\section{References}

[1] J. Lei et al., J. Struct. Biol. 150 (2005) p. 69-80.

[2] B. Carragher et al., J. Struct. Biol. 132 (2000) p. 33-45.

[3] R. M. Glaeser et al., 2006. Microsc. Microanal, 12 (suppl. 2) (2006) p. 84.

[4] J. Zhang et al., JADAS: J. Struct. Biol. 165 (2009) p. 1-9 


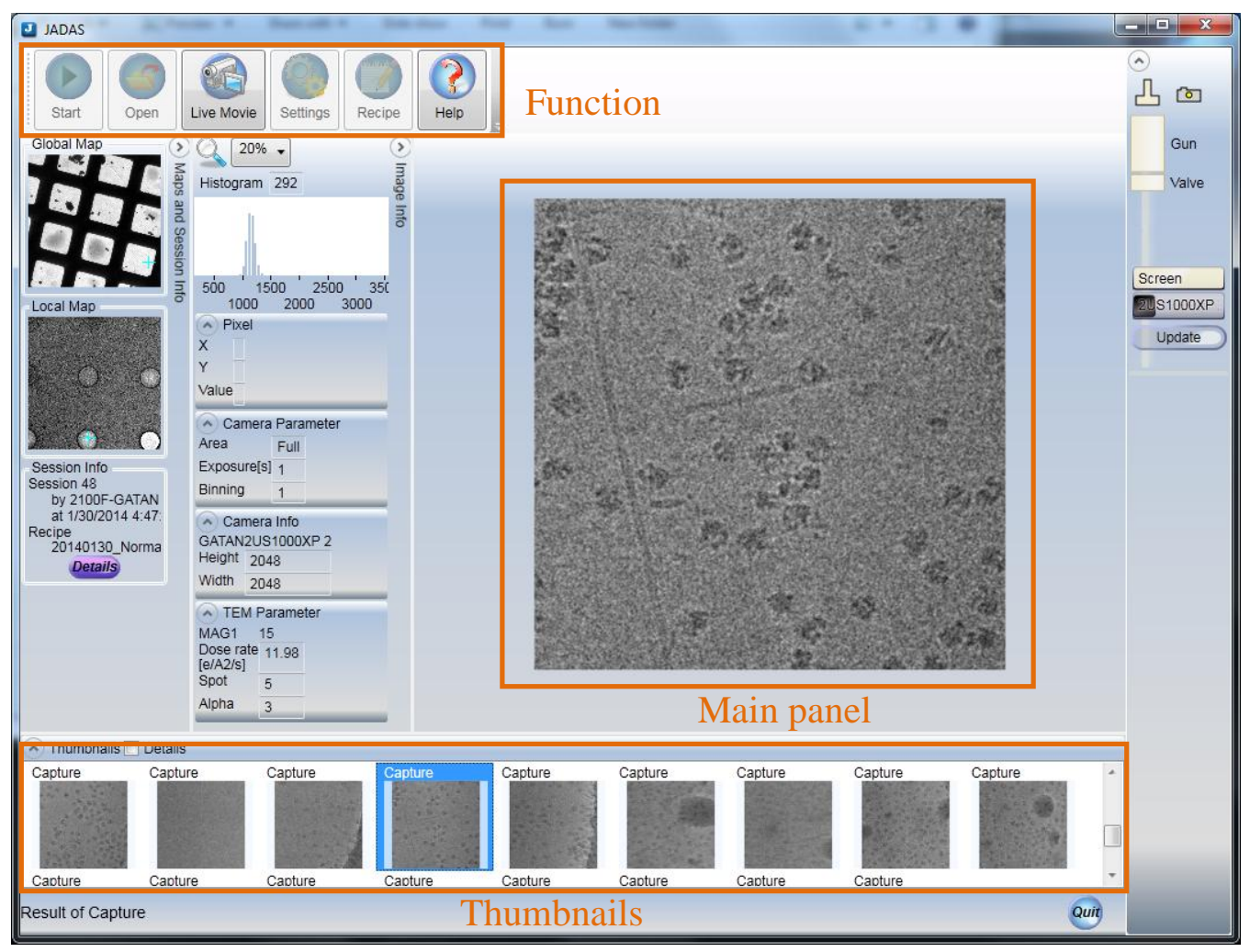

Figure 1 A screenshot of a GUI of the JADAS. Purified ribosomes were used as a sample. These images were automatically taken at $200 \mathrm{kV}$ with JEM-2100F using the new JADAS. Magnification was 60,000. Electron dose was $10 \mathrm{e}^{-/} \mathrm{A}^{2}$. 